\title{
The influence of doping on the performance of organic bulk heterojunction solar cells
}

\author{
Markus Glatthaar $^{* a}$, Nicola Mingirulli ${ }^{\mathrm{b}}$, Birger Zimmermann ${ }^{\mathrm{a}}$, Florian Clement ${ }^{\mathrm{b}}$, Moritz Riede ${ }^{\mathrm{a}}$, \\ Bas van der Wiel ${ }^{\mathrm{b}}$, Michael Niggemann ${ }^{\mathrm{a}, \mathrm{b}}$, Andreas Gombert ${ }^{\mathrm{b}}$ \\ ${ }^{a}$ Freiburger Materialforschungszentrum (FMF), Stefan-Meier-Str. 21, 79104 Freiburg, Germany \\ ${ }^{\mathrm{b}}$ Fraunhofer Institut Solare Energiesysteme (ISE), Heidenhofstr. 2, 79110 Freiburg, Germany
}

\begin{abstract}
We investigated organic bulk-heterojunction solar cells based on an absorber blend of poly(3-hexylthiophene) (P3HT) and [6,6]-phenyl C61-butyric acid methyl ester (PCBM) by electrical impedance spectroscopy (EIS). A strong neck in the modulus plot of the EIS-spectra indicates that the absorber is divided into two regions of different conductivities. A similar behaviour was observed for pure P3HT-diodes. Hence, it can be concluded that the PCBM:P3HT absorber is pdoped by impurities of P3HT, so that a Schottky-like contact with aluminium is formed. It is known from literature, that annealing of PCBM:P3HT solar cells leads to drastic improvement of the photovoltaic performance. We compared the current-voltage characteristics and impedance spectra before and after consecutive annealing steps. After the annealing an expansion of the depletion region was observed, indicating that volatile dopants were evaporated out of the absorber. This contributes to an improved photovoltaic performance as the separation of the generated charges in the depletion region is more efficient than in the non-depleted region. Also an improved rectification behaviour might be caused by a lower doping level.
\end{abstract}

Keywords: Organic solar cells, impedance, Schottky-contact, doping, annealing, post-treatment, rectification, depletion region

\section{INTRODUCTION}

The absorber of organic bulk-heterojunction solar cells is made of a blend of two different materials ${ }^{1}$. Poly(3hexylthiophene) (P3HT) and [6,6]-phenyl C61-butyric acid methyl ester (PCBM,) are among the most promising materials for such devices ${ }^{2}$. Due to the energies of the highest occupied molecular orbital (HOMO) and the lowest unoccupied molecular orbital (LUMO) of the two materials, P3HT acts after optical excitation as an electron donor, while PCBM acts as an acceptor. After the charge transfer P3HT provides an electrically conductive network to transport the hole to the anode of the solar cell. Respectively, the electron is transported on a PCBM network to the cathode. For sake of simplicity the absorber can be regarded as one effective semiconductor, where the LUMO corresponds to the conduction band edge and the HOMO to the valence band edge of an inorganic semiconductor. In this article we would like to confirm with additional experiments on pure P3HT-diodes our interpretation of the so called post-treatment on P3HT:PCBM solar cells ${ }^{3}$. We think, that the improved solar efficiency after the post-treatment of the device can not only be explained by higher absorption, higher hole mobility and the burning of shunts ${ }^{4,5}$. We found out that the P3HT:PCBM absorber can be p-doped and can forms a Schottky-contact with the aluminium electrode. We monitored the thickness of the depletion layer at the Schottky-contact by electrical impedance spectroscopy (EIS).

* markus.glatthaar@,fmf.uni-freiburg.de, phone: +49(0)761 2034782, fax: +49(0)761 2034801, www.fmf.uni-freiburg.de

Organic Optoelectronics and Photonics II, edited by Paul L. Heremans, Michele Muccini, Eric A. Meulenkamp, Proc. of SPIE Vol. 6192, 61921V, (2006) · 0277-786X/06/\$15 - doi: 10.1117/12.662695 
Annealing P3HT:PCBM solar cells under forward bias leads to significant improvement of the photovoltaic performance ${ }^{4,5}$. After such a post-treatment in consecutive steps we observed a decrease of the doping level for both, pure P3HT and P3HT:PCBM devices. This was accompanied by an increase of the rectification behaviour.

\section{EXPERIMENT}

The solar cell devices were fabricated on indium tin oxide (ITO) covered glass substrates. First an approximately $40 \mathrm{~nm}$ thick layer of poly(3,4 ethylenedioxythiophene) doped with poly(styrenesulfonate) (PEDOT:PSS) was spin coated from aqueous solution (Baytron P AI 4083, H.C. Starck). PEDOT:PSS is highly doped, so it is comparable to a metallic contact. On top the absorber blend $(350 \mathrm{~nm})$ was spin coated from chlorobenzene solution $(\sim 1 \% \mathrm{wt}$.) with a ratio of 1:1.5 wt. P3HT:PCBM (P3HT 4002 by Rieke Metals, PCBM by Nano-C). The top electrode is made of a $0.6 \mathrm{~nm}$ thick layer of lithium fluoride (LiF) followed by a $100 \mathrm{~nm}$ thick layer of aluminium (Al). Both layers were thermally evaporated under vacuum.

The P3HT diodes were also fabricated on ITO covered glass substrates but without PEDOT layer, to render more clearly that the regions of different conductivity are not given by the PEDOT:PSS and the P3HT layer. To obtain very thick layers, P3HT was spin coated from chloroform solution ( $2 \% \mathrm{wt}$.). The total layer thickness was not determined.

The impedance spectra of the devices were recorded from $1 \mathrm{~Hz}$ to $1 \mathrm{MHz}$ with a Zahner IM6 electrochemical workstation. The current-voltage-characteristic of the solar cell was measured in dark or at low illumination intensity with a halogen lamp. The low illumination intensity was used to avoid unintended heating of the samples. After preparation the devices were post-treated with two consecutive annealing steps. For the solar cells simultaneously to the annealing a forward bias voltage was applied.

\section{RESULTS AND DISCUSSION}

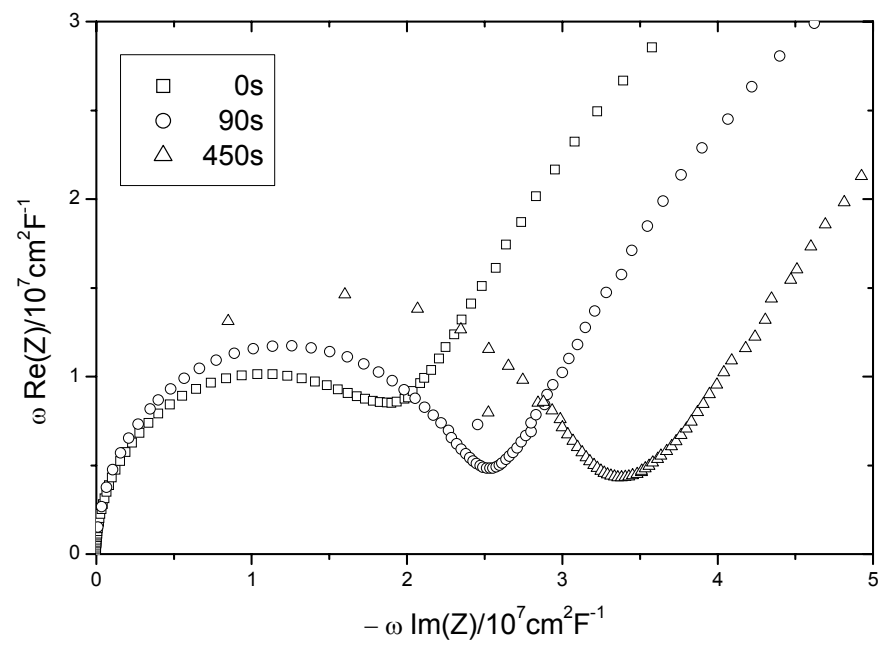

Fig. 1 Impedance spectra of a P3HT diode before and after consecutive annealing steps for $90 \mathrm{~s}$ and $450 \mathrm{~s}$ at $75^{\circ} \mathrm{C}$.

The EIS-spectra in this paper are represented by the modulus-plot. In this plot an ideal RC-element has the shape of a semi-circle, where the diameter is the reciprocal capacitance $1 / C$. Two serially connected RC-elements, $R_{I} C_{I}$ and $R_{2} C_{2}$, are still represented by a semicircle when $R_{I} C_{I}=R_{2} C_{2}$. The diameter is then the sum of the reciprocal capacitances $1 / C_{I}$ 
$+1 / C_{2}$. But the more the product $R C$ of the two elements differ, the stronger a neck occurs in the semicircle ending up in two separate semicircles. The diameter of each semi-circle then is the reciprocal capacitance of the respective RCelement. The semicircle of the RC-element with the higher resistance $R$, will occur at the left in the spectrum.

For organic bulk-heterojunction solar cells we observed a similar EIS spectrum to that of two serially connected RCelements. This indicates, that the absorber is divided into a conductive bulk region and a charge carrier depleted region at a Schottky-contact. The position of the neck moved to larger values for negative bias voltage, while it moved to smaller values for positive bias voltage ${ }^{3}$. This proves that the neck in the spectrum marks the transition from the bulk region to the depletion region. Fig.1 shows the EIS-spectra of a pure P3HT-diode before and after two consecutive annealing steps at $75^{\circ} \mathrm{C}$. The shift of the kink to larger values after the annealing hints to a reduction of the doping level. The same behaviour is observed for PCBM:P3HT solar cells after a first post-treatment for 30s with $1 \mathrm{~V}$ forward bias at $110^{\circ} \mathrm{C}$ and a subsequent treatment for $450 \mathrm{~s}$ with $2 \mathrm{~V}$ forward bias at $110^{\circ} \mathrm{C}$ (s. Fig.2).

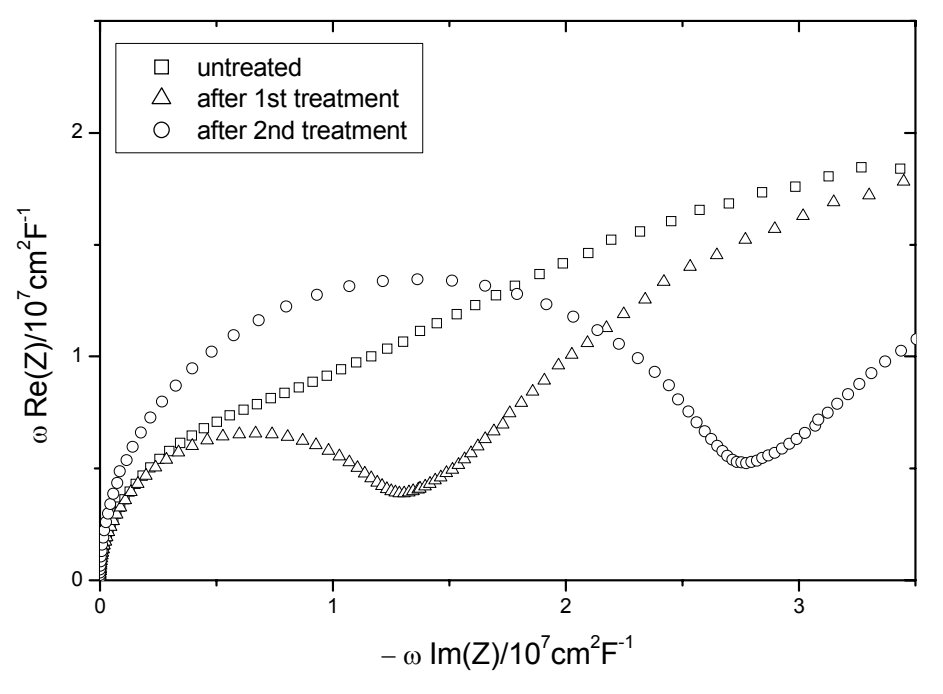

Fig.2 Impedance spectra of a PCBM:P3HT solar cell in the dark: non-treated, after a first post-treatment $\left(30 \mathrm{~s}\right.$ at $110^{\circ} \mathrm{C}$ and $1 \mathrm{~V}$ forward bias) and after the second posttreatment ( $450 \mathrm{~s}$ at $110^{\circ} \mathrm{C}$ and $2 \mathrm{~V}$ forward bias).

Both devices show an improved rectification behaviour after the treatments as can be seen in Fig.3 and Fig. 4. For solar cells it was assumed that the post-treatment causes a burning of shunts ${ }^{4}$. If these shunts were due to pinholes in the blend film, a reverse bias during the treatment should lead to better results, as the full electrical energy would be deposited selectively at the shunts. Also a stronger allocation of PCBM at the Al contact is frequently discussed. The results on the pure P3HT diode thermally treated without bias voltage give evidence for another reason for the improved diode behaviour. It is noted for organic semiconductors, that injection from a metal even at high barriers becomes possible, when the semiconductor is highly doped ${ }^{6}$. As the doping level of P3HT and PCBM:P3HT blend is reduced by annealing, the therefore lowered probability of hole injection from the Al contact could be an explanation for the improved rectification behaviour. The weak neck at the impedance spectrum of the untreated solar cell shows, that the depletion region is much more conductive than expected for a Schottky-contact ${ }^{7}$. The reason for the reduction of the doping level can be understood as a temperature enhanced vaporization of the acceptor-impurities. The applied forward bias voltage at the post-treatment of solar cells might additionally cause a drift of the impurities away from the Al contact. 


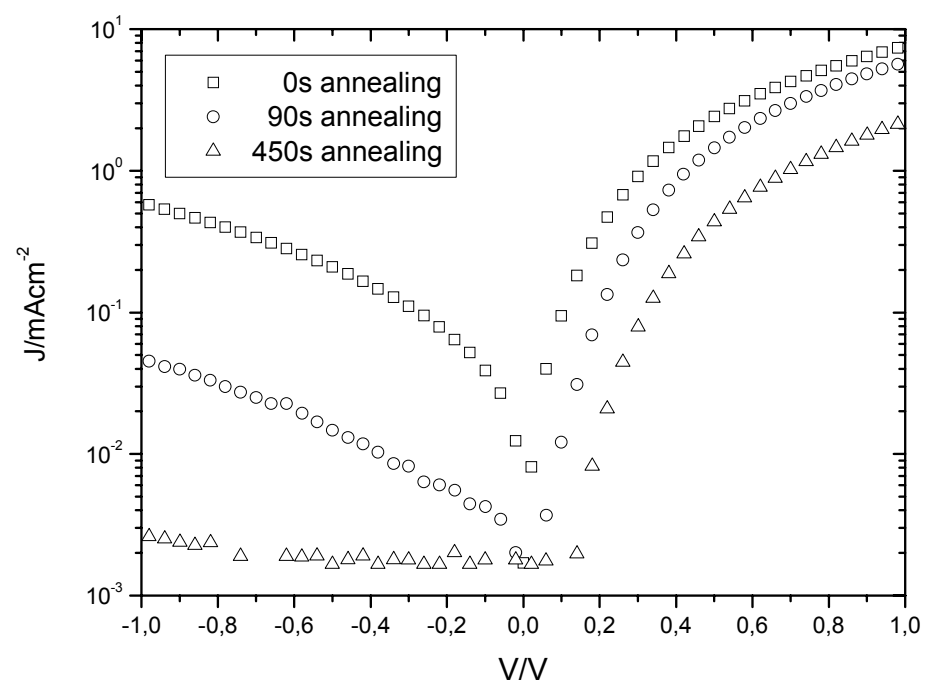

Fig. 3 Current-voltage characteristics of a P3HT diode after the two consecutive annealing steps.

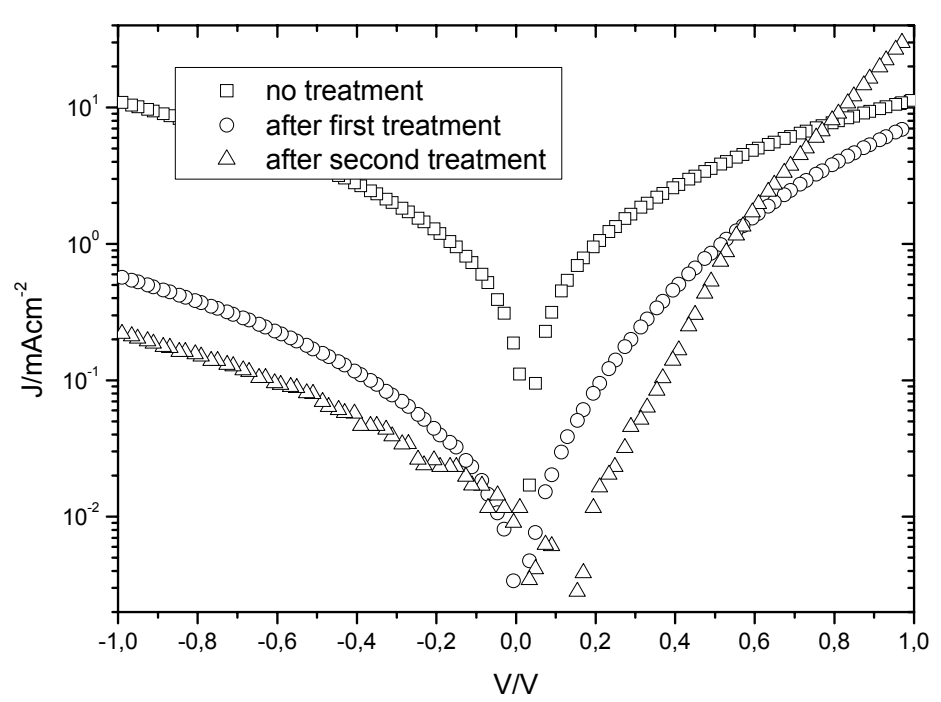

Fig. 4 Current-voltage characteristics of a P3HT diode in the dark before and after the two consecutive post-treatment steps

The effect of the post-treatment on the photovoltaic behaviour in the fourth quadrant can be seen in Fig.5. The shortcircuit current density is approximately four times higher after the second post-treatment, compared to the device after the first moderate post-treatment. The effect of the reduced doping level on the photovoltaic performance is twofold: First, it probably leads to a better rectification behaviour, which is essential in order not do shunt the photocurrent. Secondly, it leads to a larger space charge region, where the generated charges can be separated more efficiently. In 
further experiments we used purified P3HT (Rieke 4002e) and observed consistently a lower doping level and better photovoltaic performance as well as better rectification behaviour already before the post-treatment compared to devices made of unpurified P3HT. But still the post-treatment was affecting the three aspects positively.

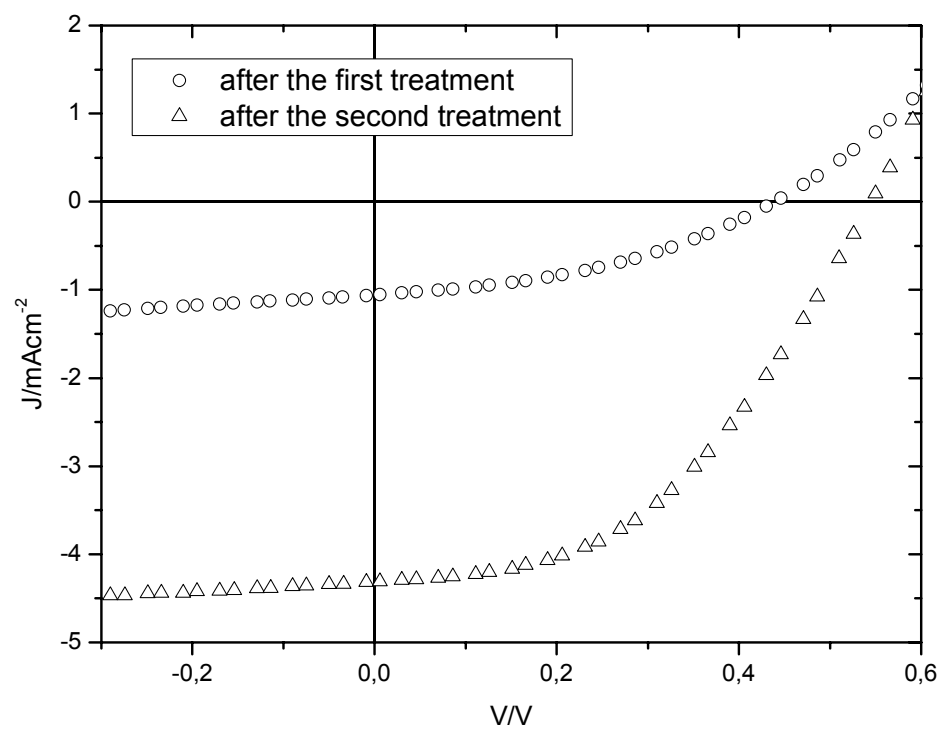

Fig. 3 Current-voltage characteristics of a PCBM:P3HT solar cell under illumination after the first and after the second post-treatment.

\section{CONCLUSIONS}

By the comparison of pure P3HT diodes with P3HT:PCBM solar cells, we were able to demonstrate that the effect of post-treatment is not specific to the absorber blend of P3HT and PCBM. By electrical impedance spectroscopy we observed that the depletion region becomes larger after the post-treatment, meaning that the doping level decreases. The rectification behaviour of the P3HT diode was improved by annealing without an applied bias voltage. We think that a reduced doping level can be a reason for this effect. The photovoltaic performance severely depends on rectification. Also the larger space charge region probably leads to a better charge separation. Using purified P3HT results in a lower doping concentration of untreated devices. But by the post-treatment the doping level can still be reduced leading to a better device performance.

\section{ACKNOWLEDGEMENTS}

This work was supported by the European Union Project Molycell, project-Nr. SES6-CT-3003-502783. 


\section{REFERENCES}

1. C. Brabec, N. Sariciftci, C. Hummelen, Adv. Funct. Mater. 2001, 11, No. 1

2. V. Dyakonov, Appl. Phys. A 79, 21 (2004)

3. M. Glatthaar, N. Mingirulli, B. Zimmermann, T. Ziegler, R. Kern, M. Niggemann, A. Hinsch, A. Gombert, phys. stat. sol., 202, 11 (2005), p. R125-R127

4. F. Padinger, R.S. Rittberger, N.S. Sariciftci, Adv. Func. Mat. 13, 85 (2003)

5. Y. Kim, S.A. Choulis, J. Nelson, D.D.C. Bradley, S. Cook, J.R. Durrant, Appl. Phys. Lett. 86, 063502 (2005)

6. A. R. Hosseini, M.H. Wong, Y. Shen, G.G. Malliaras, J. Appl. Phys. 97, 023705 (2005)

7. J. Singh, Semiconductor Devices - Basic Principles (Wiley \& Sons, New York, 2001), p. 238 